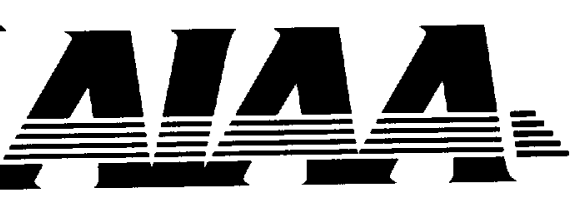

\title{
AIAA 2000-4009 \\ Prediction of Hyper-X Stage Separation Aerodynamics Using CFD
}

Pieter G. Buning

NASA Langley Research Center Hampton, VA

\author{
Tin-Chee Wong, \\ Arthur D. Dilley, \\ Jenn L. Pao \\ FDC/NYMA, Inc. \\ Hampton, VA
}

\section{AIAA 18th Applied Aerodynamics Conference August 14-17, 2000 / Denver, CO}


AIAA 2000-4009

\title{
PREDICTION OF HYPER-X STAGE SEPARATION AERODYNAMICS USING CFD
}

\author{
Pieter G. Buning* \\ NASA Langley Research Center, Hampton, VA \\ Tin-Chee Wong, Arthur D. Dilley, and Jenn L. Pao \\ FDC/NYMA, Inc., Hampton, VA
}

\begin{abstract}
The NASA X-43 "Hyper-X" hypersonic research vehicle will be boosted to a Mach 7 flight test condition mounted on the nose of an Orbital Sciences Pegasus launch vehicle. The separation of the research vehicle from the Pegasus presents some unique aerodynamic problems, for which computational fluid dynamics has played a role in the analysis. This paper describes the use of several CFD methods for investigating the aerodynamics of the research and launch vehicles in close proximity. Specifically addressed are unsteady effects, aerodynamic database extrapolation, and differences between wind tunnel and flight environments.
\end{abstract}

\section{Introduction}

The Hyper-X research program was initiated in 1996 to demonstrate in-flight hypersonic scramjet propulsion. To get to the flight test conditions, the $12 \mathrm{ft}$ long research vehicle (HXRV) is mounted on the nose of the first stage of an Orbital Sciences Corporation Pegasus booster. Given the non-axisymmetric shape of the HXRV, it is mounted onto an adapter that covers the external nozzle (aft undersurface) of the research vehicle (Fig. 1). This configuration, referred to as the Hyper-X Launch Vehicle (HXLV), is carried aloft by a NASA B-52. After release from the B-52, the Pegasus boosts the research vehicle to a nominal scramjet test condition of Mach 7 and $95,000 \mathrm{ft}$ altitude, at a dynamic pressure of $1,000 \mathrm{lb} / \mathrm{ft}^{2}$ and Reynolds number of 0.94 million per foot. Separation between the booster and research vehicle is accomplished with ejection pistons, pushing the HXRV with a stroke of 9 inches in $100 \mathrm{~ms}$; the research vehicle clears the front of the adapter in $250 \mathrm{~ms}$. $^{1}$

*Associate Fellow, AIAA.

Copyright (C) 2000 by the American Institute of Aeronautics and Astronautics, Inc. No copyright is asserted in the United States under Title 17, U.S. Code. The U.S. Government has a royalty-free license to exercise all rights under the copyright claimed herein for Governmental purposes. All other rights are reserved by the copyright owner.

\section{Wind Tunnel Program}

A wind tunnel test program was undertaken to define the stage separation aerodynamic environment. ${ }^{2}$ Preliminary separation concepts were tested in the NASA Langley 20" Mach 6 tunnel, but the majority of the force and moment data used in the separation aerodynamic database was obtained from a test in the Arnold Engineering Development Center (AEDC) von Karman Facility Tunnel B, using the Captive Trajectory System (CTS) rig. This system allowed the relative positioning of the research vehicle and the booster, with force data available from each. Tunnel flow conditions were Mach 6, with a static temperature of $104^{\circ} \mathrm{R}$ and test Reynolds number of 2.2 million per foot. The model was $1 / 12^{\text {th }}$ scale. Boundary layer trips were employed on the research vehicle nose, adapter, and booster fuselage and aerodynamic surfaces.

While the current configuration of interest has an adapter that remains fixed to the booster, another approach was also investigated during the AEDC wind tunnel test. This concept involved rotating the adapter downward to help increase clearance with the research vehicle during separation and reduce aerodynamic interference. While this "drop-jaw" approach was not adopted for the flight vehicle, it did occupy a significant amount of resources during the test, reducing the number of test points on the basic configuration with the fixed adapter. In addition, while use of the CTS rig vastly improved productivity of the tunnel in gathering data over a range of separation distances, it also imposed a limit in "closest approach" of the two vehicles, to avoid the possibility of contact. This had the effect of limiting the ability to test some of the most critical relative positions, where the most interference would be expected during the separation maneuver. Since the AEDC test, additional separation data has been obtained from tests in the Langley 20" Mach 6 tunnel, though the $A E D C$ data remains the primary source for the stage separation aerodynamic database. 


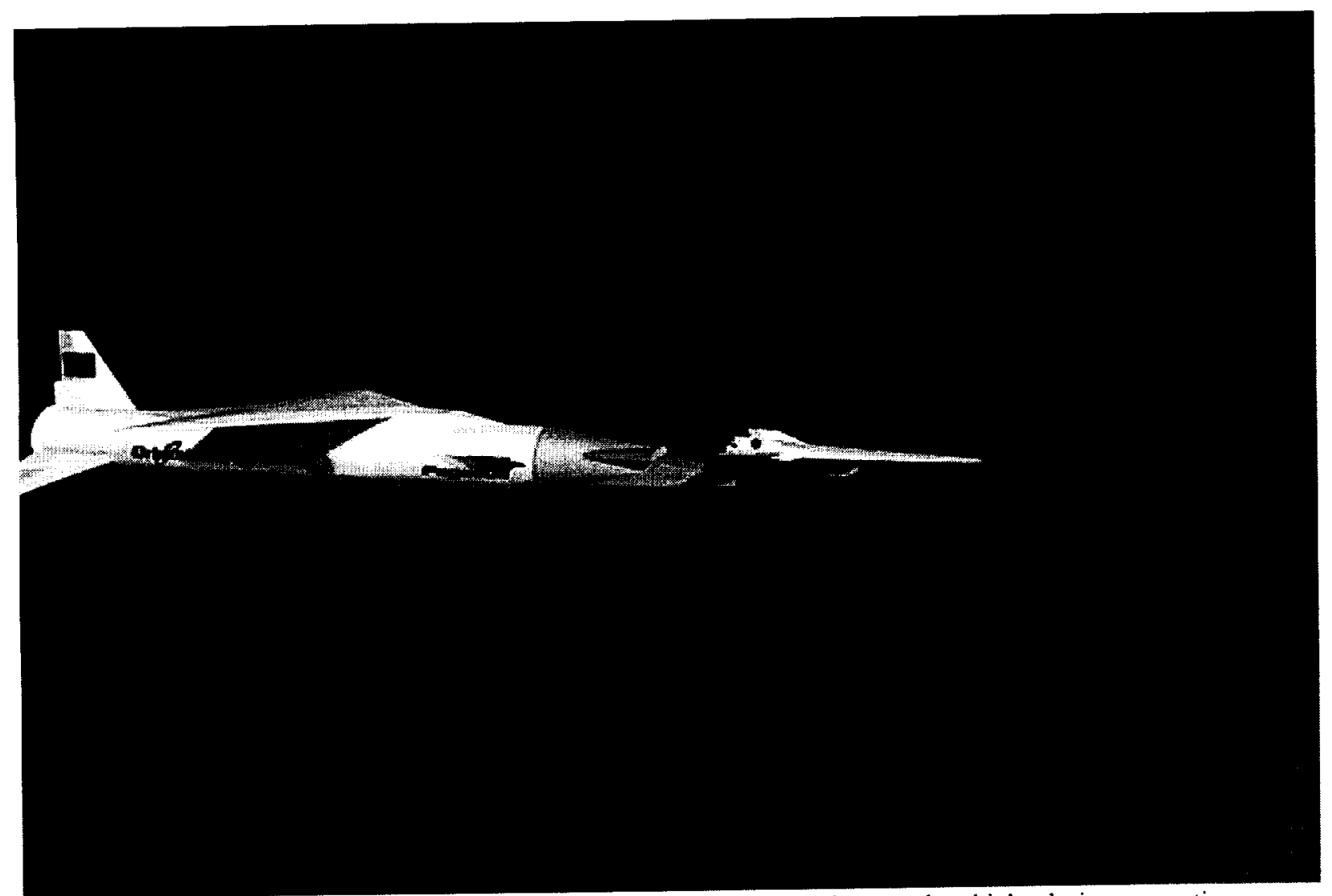

Fig. 1. Hyper-X launch vehicle consisting of booster, adapter and research vehicle, during separation.

\section{Pre-Test CFD}

Prior to the AEDC wind tunnel test, CFD calculations were used to investigate the drop-jaw concept. These inviscid flow simulations modeled the jaw rotated downward at several different angles, and helped identify aerodynamic problems with the concept that were investigated further in the AEDC test. Good agreement in trends and order-of-magnitude variation in force and moments was obtained, though absolute levels could not be matched due to the viscous nature of the flow between the adapter and research vehicle. Though not part of the focus of this paper, analysis of the drop-jaw concept contributed to design choices for the stage separation, and provided an opportunity to identify some capabilities and limitations of inviscid simulation for hypersonic multibody flows.

\section{Aerodynamic Issues}

Even following the AEDC test, several aerodynamic issues remained in fully understanding the dynamics of the stage separation maneuver. This understanding was complicated by the unsteady nature of the event, the number of degrees of freedom associated with the booster, research vehicle, and control surfaces, and limits in the amount of wind tunnel data available. These issues were in three basic areas: unsteady effects, aerodynamic database extrapolation, and differences between wind tunnel and flight conditions.

Two types of unsteady aerodynamic effects occur during the separation. The first is the initial separation transient, caused by the establishment of quasi-steady flow in the gap that opens up between the research vehicle and the adapter. The second is the unsteady aerodynamic component due to the general dynamics of the separation maneuver.

The database used to define the aerodynamic coefficients of the research vehicle during the separation maneuver consists of three flow parameters $(\alpha, \beta, M a c h)$, six separation parameters (translations Xsep, Ysep, Zsep, and Euler angles Asep, Bsep, Csep), and three control surface deflections. ${ }^{4}$ The piston travel during the first 9 inches of separation imposes constraints on the initial part of the separation, but significant regions of the database have no AEDC wind tunnel data to define the aerodynamic coefficients, due to the previously mentioned constraint on "closest approach" imposed by use of the CTS rig. Further, normal force and pitching moment on the research vehicle change greatly depending on whether the adapter is masked or exposed to the oncoming highspeed flow. 
Finally, a number of differences exist between the wind tunnel and flight environments, including model mounting interference, freestream Mach and Reynolds number, geometry of the adapter seal next to the research vehicle, whether the upper surface flow is laminar or turbulent, and adiabatic vs. isothermal behavior of the exposed surfaces.

\section{CFD Approaches}

The following sections will present some examples of how computational fluid dynamic (CFD) modeling has contributed to a better understanding of these aerodynamic issues. Several computational approaches have been used in these studies, due to suitability and availability of codes, and experience of the researchers involved. These include viscous and inviscid analyses using overset structured and unstructured grid flow solvers. These analyses are concerned only with generating estimates of aerodynamic forces, not heating information. Force and moment estimates in the aerodynamic database are used as inputs to the multibody stage separation trajectory simulations. All CFD simulations assume a perfect gas with a constant specific heat ratio of 1.4 , adequate for modeling Mach 6 flow. Further documentation on CFD methodology used for various aspects of the Hyper-X program can be found in Ref. 5.

\section{Computational Tools}

Two different Euler/Navier-Stokes flow solvers have been used for these studies. These are SAMcfd, ${ }^{6.7}$ for drop-jaw simulations prior to the AEDC wind tunnel test, two-dimensional time-accurate analyses of initial flow establishment through the gap between the research vehicle and adapter, and 3D stage separation simulations; and OVERFLOW, ${ }^{8.9}$ for viscous simulation of steady flow about a number of separation configurations. OVERFLOW is a structured, overset grid Navier-Stokes flow solver, developed at NASA, and SAMcfd is an unstructured grid CFD package under development by ResearchSouth, Inc., and includes flow solver and grid generation capability.

The Hyper-X research vehicle has undergone several geometry revisions. The geometry used for these simulations represents the keel-line 4 definition, except for the pre-wind tunnel-test cases, which used keel-line 3.

The advancing front grid generation capability within SAMcfd was used for the inviscid flow cases. This capability was particularly suited to the stage separation problem because of its ease in manipulating the multiple body geometry, and straightforward gridding of complex shapes. Grids with 4 to 5 million cells were generated for the stage separation configurations, and 5000 iterations were used to reach steady state. Calculations were performed on the NASA NAS Cray C-90.

For the overset grid system used by OVERFLOW, the Chimera Grid Tools package ${ }^{10}$ was used to generate surface patches covering the surface of the vehicle and adapter, then body-fitted volume grids from these surfaces. These volume grids were buried in a cartesian background grid, sized to include the research vehicle (or "flyer") and adapter in their specified relative positions (Fig. 2). For the various stage separation configurations, overset grids are seen as an advantage for the grid generation process, as body-fitted grids associated with the moving component(s) are simply translated and rotated to their new location, and the grid joining process is executed to reconnect the body-fitted and background grids. For this effort, the PEGSUS 4 code $^{11}$ was used to connect the overset grids.

Computational grids were constructed in units of full-scale inches, and wind tunnel scale effects were incorporated by using an appropriate Reynolds number. In these units, initial grid spacing off the surface was set at 0.001 inches. This was based on a turbulent boundary layer $\mathrm{y}^{+}$value of 1 at a downstream distance of 2 inches (10\% of a fin tip chord), using a flat plate correlation method from White ${ }^{12}$ for the wind tunnel Reynolds number. A more sophisticated model of Sommer and Short ${ }^{13}$ incorporates compressibility as a function of freestream temperature and Mach number. Use of this model significantly relaxes the grid spacing requirement, resulting in a minimum spacing of 0.02 " for wind tunnel conditions, and 0.005 " for flight. As a result, the 0.001 " spacing was used for all turbulent cases, and was adequate for computing laminar cases as well. Examination of computed turbulent solutions confirmed the Sommer and Short estimates, and laminar solutions showed 20 to 25 grid points in the boundary layer.

The research vehicle and adapter were covered with overlapping surface grids that resolved various geometry features. Fin leading and trailing edges were covered with wraparound strips, as were the vehicle and engine inlet leading edges. Body-fitted volume grids were grown to a distance of 8 inches off the surface. Grid size was 1.4 million points for the research vehicle (including wind tunnel blade support hardware), and 0.6 million for the adapter. The background grid was 0.8 million points, for a total of 2.8 million points in 52 component grids. In order to establish the low base pressure flow fields, a sequence of runs were made for each case, starting with high smoothing levels and small time steps. Central spatial differencing was used for the flow equations, and the Spalart-Allmaras turbulence model ${ }^{14}$ was employed for turbulent flow cases. Grid sequencing and multigrid were used to accelerate steady state convergence, and converged forces and moments were obtained after 2000 to 4000 


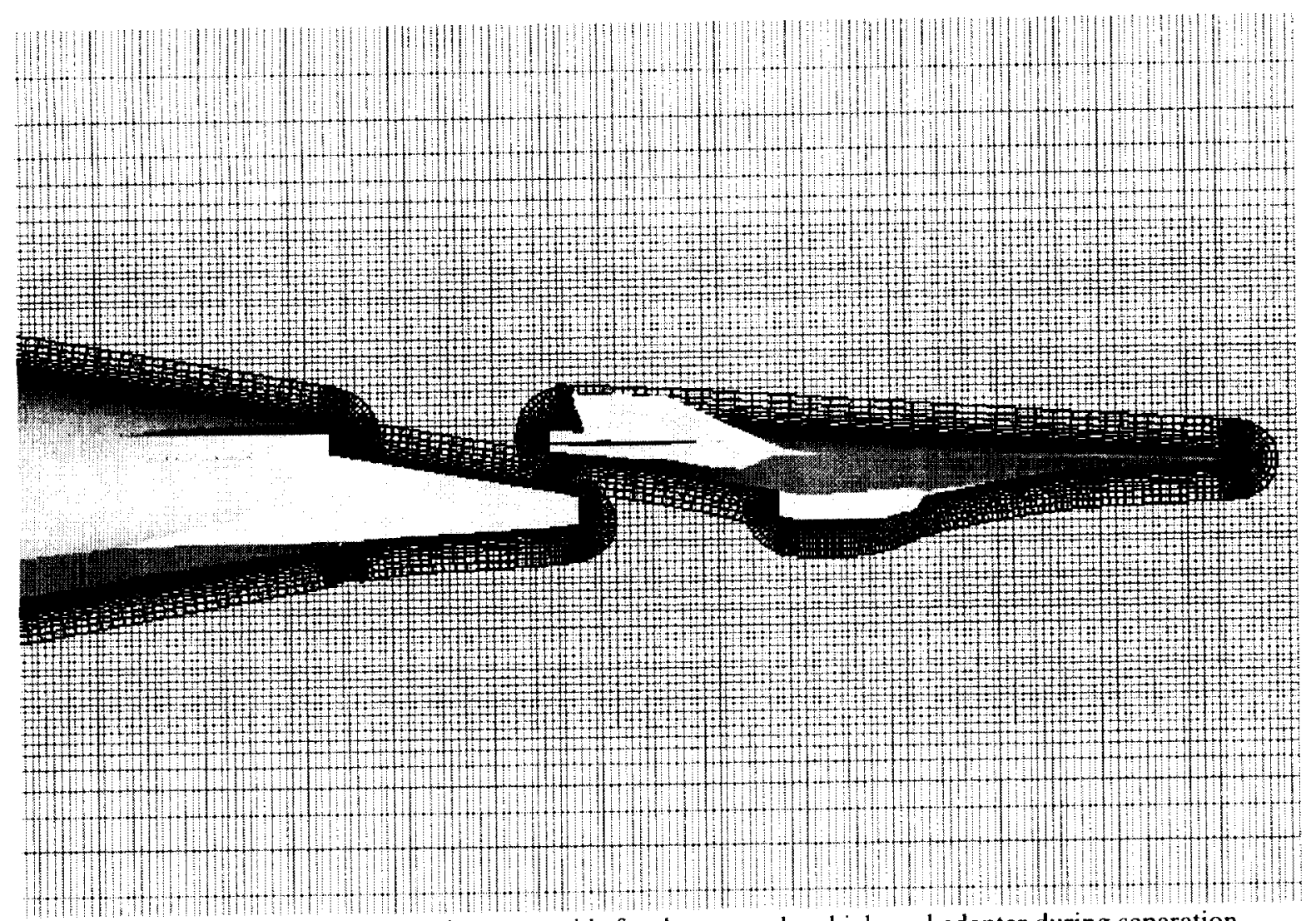

Fig. 2. Symmetry plane of overset grids for the research vehicle and adapter during scparation.

steps on the fine grid level. Various OVERFLOW computations were run on the NAS Cray C-90 and SGI Origin 2000, the DoD NAVO Major Shared Resource Center Cray SV1, and an SGI Indigo ${ }^{2}$ workstation.

\section{Validation of Grid and Approach}

In order to validate the use of OVERFLOW for high Mach number flows and gain confidence in the adequacy of the grid system developed for the stage separation configuration, a series of simulations were made of the HXRV alone, and compared to existing free flyer (HXRV) wind tunnel data from test T6776. ${ }^{15}$ The wind tunnel model was a sting-mounted $1 / 8^{\text {th }}$ scale model, tested in the Langley 20" Mach 6 tunnel, without boundary layer trips. The computations assumed laminar flow with adiabatic walls. No sting was included in the computations. Normal force, axial force, and pitching moment coefficients $\left(\mathrm{C}_{N}, \mathrm{C}_{\mathrm{A}}\right.$, and $\mathrm{C}_{\mathrm{m}}$, resp.) are plotted in Fig. 3. Agreement in normal force and pitching moment is quite good, and axial force agreement is fair over a large range of angle-ofattack $(\alpha)$. The target angle-of-attack for stage separation is 0 , while the research vehicle engine test condition is $\alpha=2^{\circ}$. This level of agreement was deemed adequate for computations of the separation configurations. Also plotted on Fig. 3 are results of one run at wind tunnel conditions with turbulent boundary layers, and one run at flight Reynolds number with wall temperature held at $1,500^{\circ} \mathrm{R}$. Normal force and pitching moment agree closely with the wind tunnel measurements, with axial force altered as expected.

Inviscid simulations at $\alpha=0$ and $2^{\circ}$ using the SAMcfd flow solver yielded a similar level of agreement in normal force and pitching moment.

\section{Unsteady Effects}

Given confidence in the solvers to compute flows about the research vehicle at Mach 6, and in the grid systems to resolve the flow adequately to capture aerodynamic forces and moments, interest shifted to specific remaining aerodynamic issues of the stage separation maneuver. With the availability of a certain amount of steady-state wind tunnel data from AEDC, attention was focused on the unsteady aspects of the stage separation. Two areas were identified and investigated.

\section{Initial Transient}

The first issue was the transient at the initial separation. Due to the geometry of the mated vehicle, it is possible for the pressure in the gap between the adapter and the aft underside of the flyer to be significantly different than the value for a steady-state 

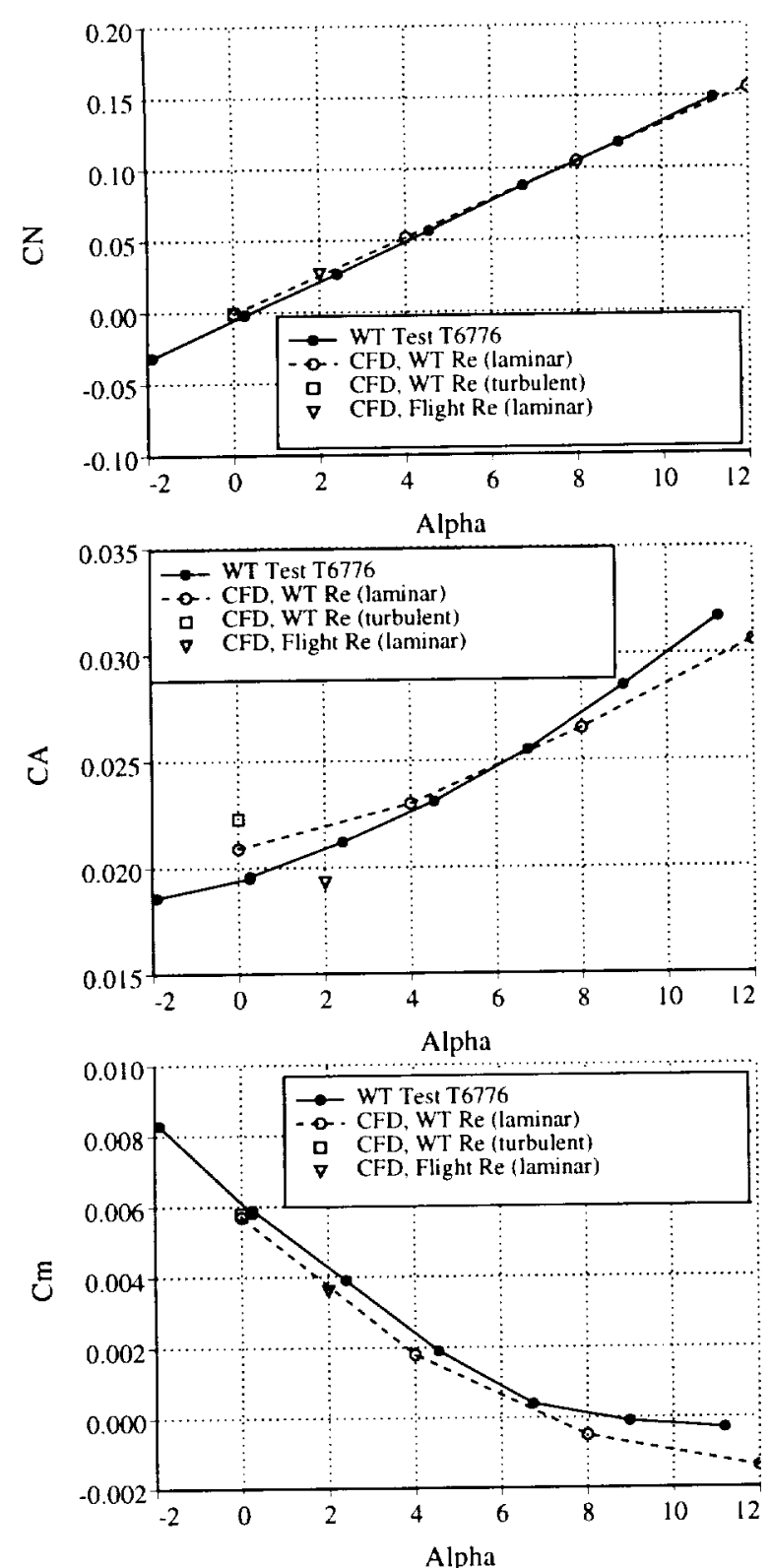

Fig. 3. HXRV (flyer-alone) CFD-calculated normal force, axial force and pitching moment compared to wind tunnel data.

flow shortly after separation. When the vehicles separate, this pressure will equalize with the external flow, but may provide an initial "kick" in the normal force and pitching moment. The speed at which this pressure equalizes relative to the overall separation maneuver determines how significant this kick is to the trajectory of the separation.

To investigate this process, a somewhat different scenario was investigated. A two-dimensional, timeaccurate simulation was carried out using the centerline geometry of the flyer and adapter, at separation distances of 0.5 and 2 inches ${ }^{16}$ (nominally 44 and $60 \mathrm{~ms}$ from separation, resp.). In this $2 \mathrm{D}$ case the gap flow was significantly more restricted than in the full 3D geometry, being driven by the flow at the adapter lip and venting at the flyer base (Fig. 4). External flow was converged with the gap sealed, and the gap flow was initialized to very low Mach number and freestream static pressure. The transient response was measured starting with the removal of the gap seals. This analysis was done first using the unstructured solver SAMcfd. As seen in Fig. 5, forces in the gap region stabilized in less than $10 \mathrm{~ms}$ in both cases. A viscous flow simulation was done using the CFL3D structured grid flow solver ${ }^{17}$ for the 2 " separation case. Again the flyer forces stabilized in less than $10 \mathrm{~ms}^{18}$ This length of time is short compared to the overall separation event, and in fact short compared to the time on the ejection pistons, during which the relative motion of the flyer is constrained. Further, these twodimensional simulations are considered very conservative, given that the real geometry provides pressure relief along the sides of the gap, resulting in a steady-state pressure close to free stream. Thus the

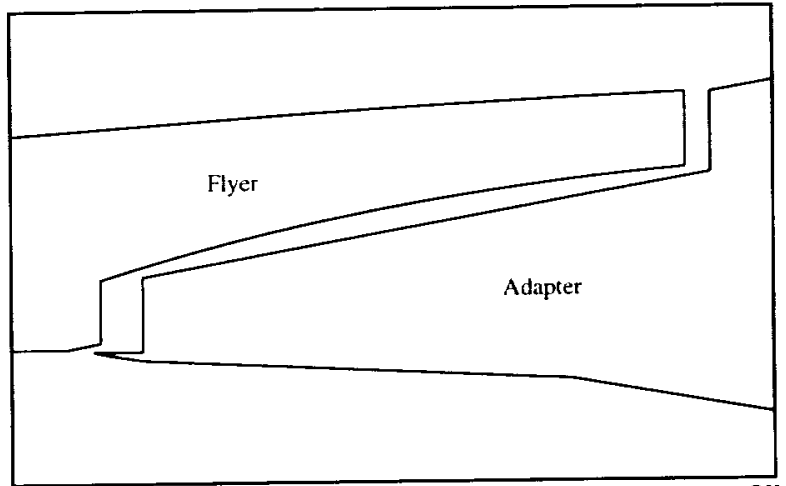

Fig. 4. Centerline flyer/adapter gap geometry at 2" separation.

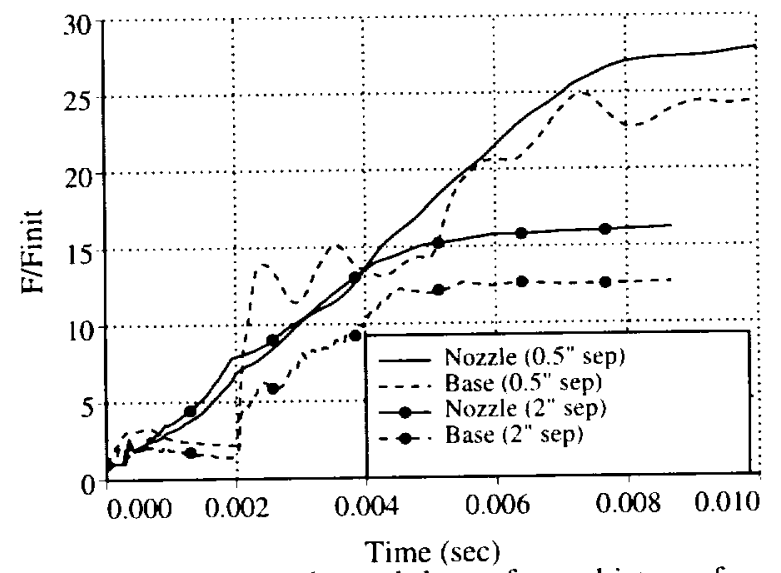

Fig. 5. Flyer nozzle and base force history from inviscid 2D simulations at 0.5 " and 2" separation (from Ref. 16). 
flow establishment time for the initial separation transient is not considered a problem.

\section{Separation Dynamics}

The second issue related to unsteady effects is the general unsteady nature of the dynamic separation process, in other words, how the fact that the booster and flyer are moving relative to each other affects the aerodynamics of the separation. The general problem of using CFD to simulate time-accurate store separation has been studied, and a number of moving body applications in transonic flow have been computed using a version of OVERFLOW which incorporates six degree-of-freedom dynamics in response to aerodynamic forces. ${ }^{19-21}$ In fact, CFD simulations of stage separation for similar vehicles have been reported previously. $^{22.23}$ While one original goal of this work was to run a time-accurate simulation of the dynamic separation, the following analysis indicates that this is not necessary. At flight conditions, the vehicle velocity at stage separation is approximately $7,000 \mathrm{ft} / \mathrm{sec}$. The relative speed between the booster and research vehicle at 4 inches separation (Xsep $=-4$ ") is $14 \mathrm{ft} / \mathrm{sec}$, or $0.2 \%$ of the free stream. This makes unsteady effects almost immeasurable compared to the steady aerodynamics. At 44 inches separation, the relative speed is still only $20 \mathrm{ft} / \mathrm{sec}$. At this speed, the flyer travels 2.4 body lengths for every added inch of separation, easily adequate to establish quasi-steady flow.

One simulation was performed with OVERFLOW at Xsep $=-4$ ", with an added forward velocity on the flyer of $6 \mathrm{ft} / \mathrm{sec}$ (scaled for a Mach 6 wind tunnel freestream velocity of $3,000 \mathrm{ft} / \mathrm{sec}$ ). This calculation showed differences in normal force, axial force, and pitching moment coefficients on the order of $0.4 \%$, similar to the convergence accuracy of the steady-state simulations. Differences on this order can be expected based solely on the change in forward speed of the flyer, e.g., the change in dynamic pressure $Q_{\text {unsteady }} / Q_{\text {ref }}=\left[\left(V_{\text {ref }}+V_{\text {sep }}\right) / V_{\text {ref }}\right]^{2}$ (where for the wind tunnel conditions, $V_{\text {ref }}=3,000 \mathrm{ft} / \mathrm{sec}$ and $V_{\text {sep }}=6 \mathrm{ft} / \mathrm{sec}$ ). Thus from past experience, and demonstrated by this analysis, the assumption of quasi-steady flow for the separation maneuver is justified.

\section{Aerodynamic Database Enrichment}

Perhaps the most significant contribution of CFD to the Hyper-X separation aerodynamics is in understanding the development and application of the aerodynamic database of force and moment coefficients. As mentioned, the database is currently based on wind tunnel data alone, and has 12 parameters: three flow conditions, six separation geometry parameters, and three control surface deflections. Concentrating on the separation geometry parameters alone, and in the longitudinal direction, we will examine the variation of normal force, axial force, and pitching moment coefficients $\left(C_{N}, C_{A}\right.$, and $C_{m}$, resp.) with Xsep, Zsep and Asep. Xsep and Zsep are defined as the horizontal and vertical offsets between the flyer and the adapter, with Xsep and Zsep positive with flyer motion aft and up, respectively (Fig. 6). Asep is the relative rotation of the booster about its reference center, which is some 250 inches aft of the adapter lip (leading edge). Asep is measured in degrees, with a nose-up rotation being positive. ${ }^{24}$

CFD simulations modeled the flyer and adapter, and included the blade support of the flyer. For the stage separation wind tunnel tests, the boundary layer was tripped on both the flyer and the adapter; CFD calculations were run fully turbulent. Several cases were run corresponding to available wind tunnel data, and results are plotted in Fig. 7. Though variations are small, agreement for these cases is excellent.

Based on dynamic trajectory simulations, the initial separation trajectory shows the flyer moving forward and down along (but not touching) the adapter top surface. This is due to the values of the predicted aerodynamic coefficients, and that the ejector pistons push against the base of the flyer in a somewhat downward direction so as to push through the flyer center of gravity. As a result, the research vehicle travels almost exclusively through regions of the aerodynamic database for which there is no AEDC wind tunnel data, due to the minimum approach limitations of the CTS rig. The trajectory simulations of the separation maneuver therefore use extrapolated data from that test.

An examination of the aerodynamic coefficients used in the extrapolation shows highly nonlinear behavior in Zsep and Asep (shown in Fig. 8, with additional data from test T6788 in the NASA 20 " Mach 6 tunnel $^{2}$ ). This is due to the effect that with increased vertical clearance, the adapter lip emerges from the

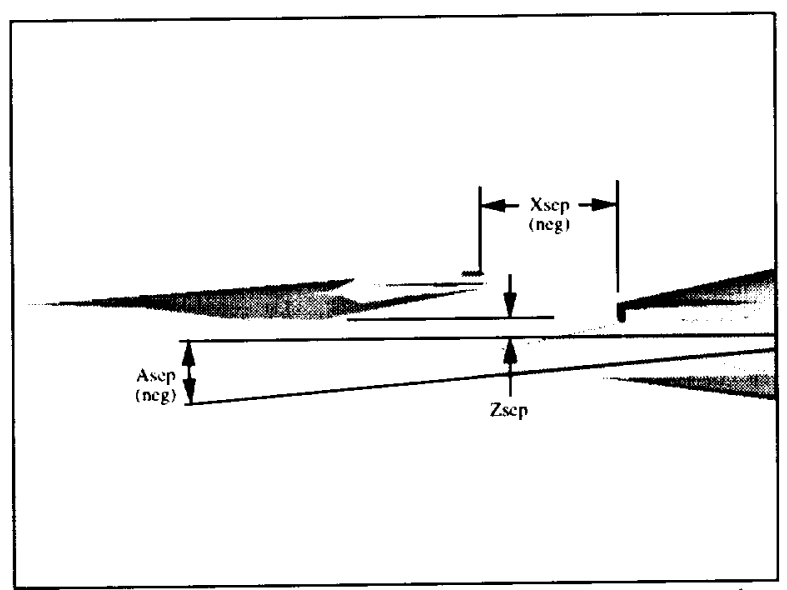

Fig. 6. Definition of longitudinal separation parameters Xsep and Zsep (translations), and angle Asep. 

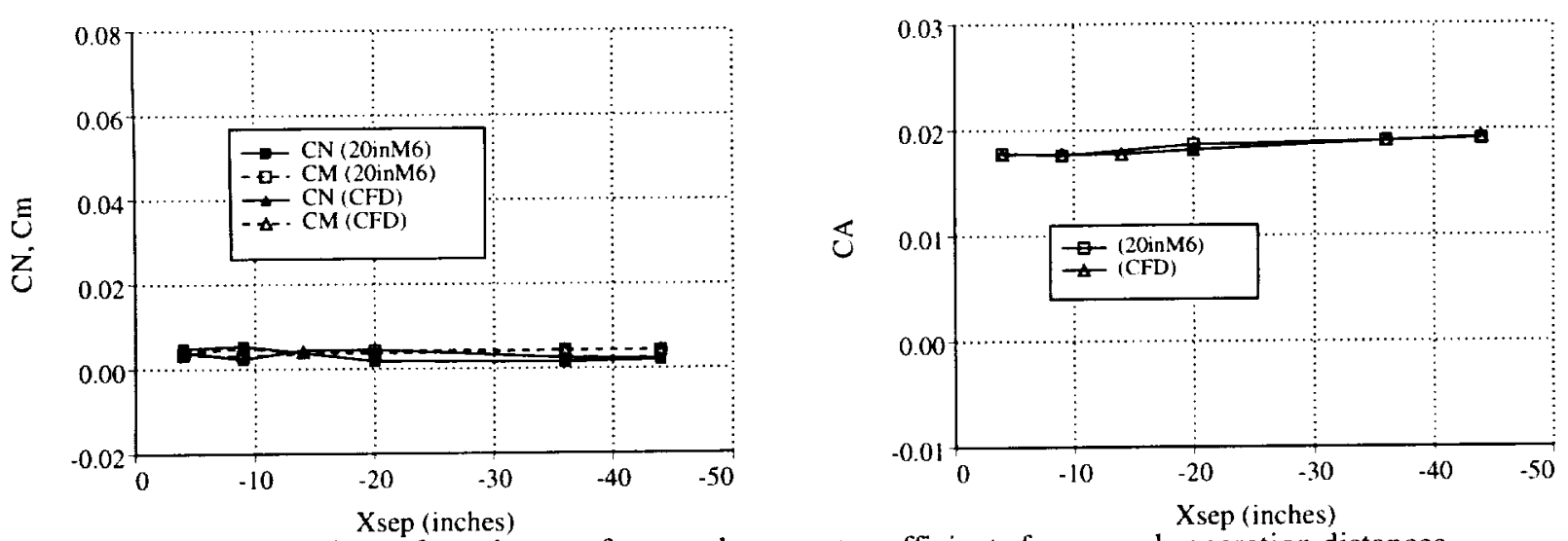

Fig. 7. Comparison of steady-state force and moment coefficients for several separation distances.

wake of the flyer and is exposed to high velocity flow. This creates a strong bow shock, which interacts with the research vehicle wake to pressurize the HXRV underside, causing a large increase in normal force and nose-down pitching moment. Fig. 9 illustrates this with an image of pressure on the underside of the research vehicle, for Asep of 0 and $-1^{\circ}$ at Xsep=-4". Here the pressure increase in the engine exit region with increased vertical separation is clearly visible. From the wind tunnel data in Fig. 8 alone, it is difficult to arrive at consistent trends in order to extrapolate for reduced vertical clearance (negative Zsep values or positive Asep angles).

Due to the sparse amount of tunnel data when considering both Zsep and Asep variations, an approach has been developed for transforming Asep and Zsep into an equivalent Zsep, which measures the effective adapter lip-to-flyer vertical separation. Geometrically, a one-degree rotation in Asep results in a 4.4-inch vertical motion of the adapter lip, due to the far aft location of the rotation point (see Fig. 6). Accordingly, we define $Z_{\text {sep }}$ equir $=Z$ sep-4.4Asep, where Zsep is in inches and Asep in degrees. With this technique, data with variations in Asep and/or Zsep can be plotted together, showing the effect of vertical separation on the aerodynamic coefficients. Data from Fig. 8 is replotted in Fig. 10 vs. Zsepequiv, and now shows general agreement between the AEDC and 20" Mach 6 tunnel data. Further, the 20" Mach 6 data indicates that for equivalent Zsep less than 2", the trend of all three coefficients is relatively flat, in contrast to the last available data from AEDC. (Again, the aerodynamic database uses AEDC wind tunnel data; linear extrapolation is used in Asep and Zsep.) For large vertical separation, force and moment coefficients approach values for the research vehicle alone.
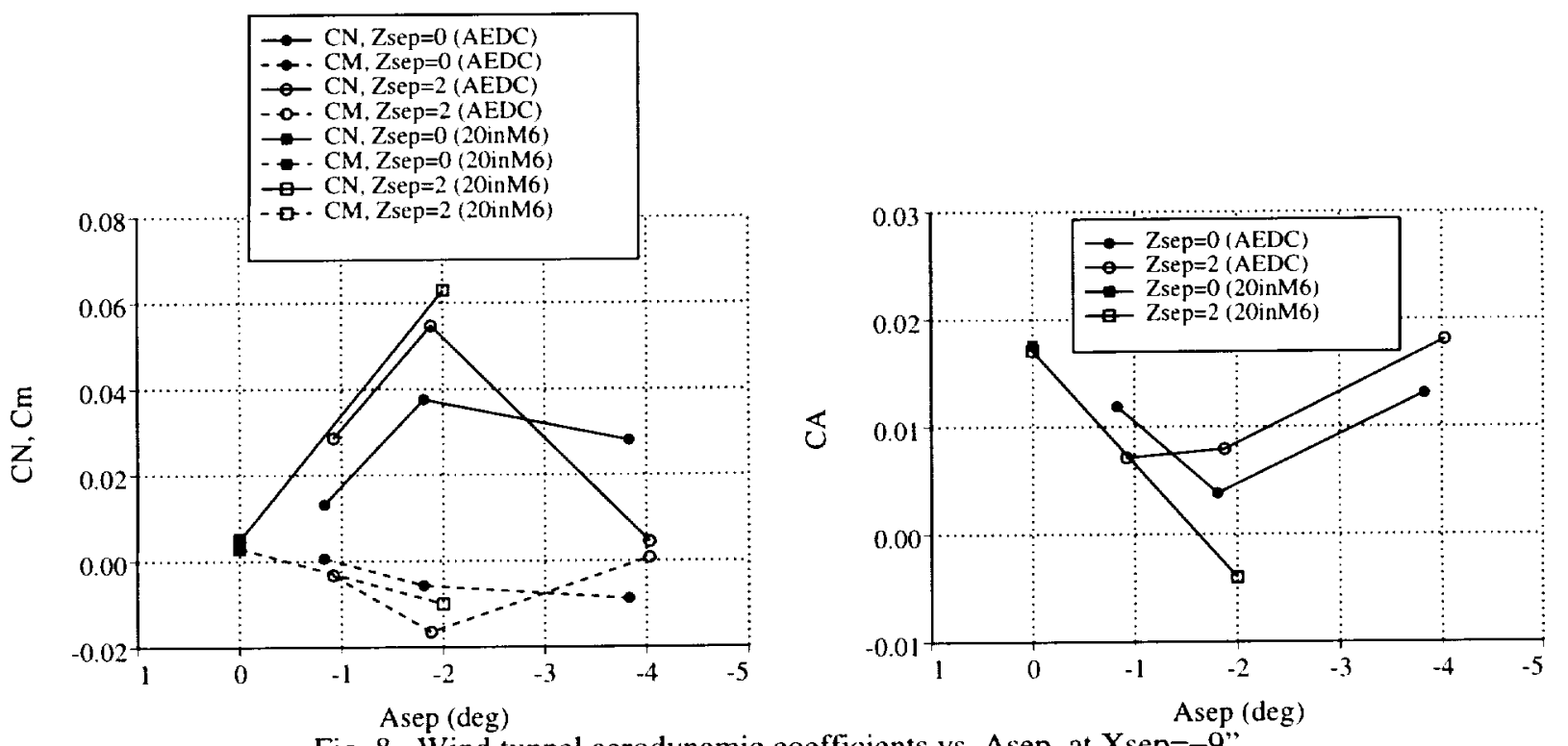

Fig. 8. Wind tunnel aerodynamic coefficients vs. Asep, at Xsep=-9". 


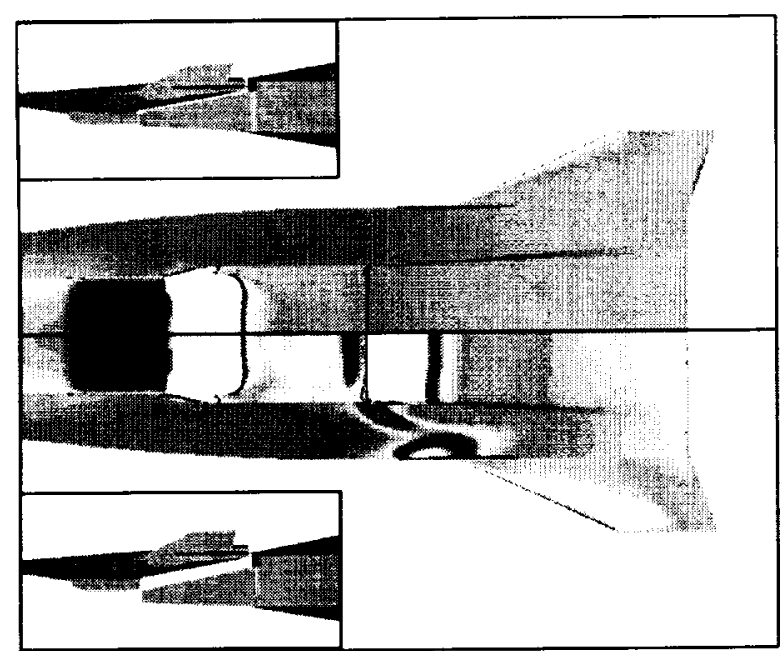

Fig. 9. Pressure on the underside of the research vehicle at $A s e p=0$ (top) and $-1^{\circ}$ (bottom), at $X$ sep $=-4$ " and Zsep $=0$.

In order to further examine the behavior of the coefficients in regions of reduced vertical separation, CFD runs with OVERFLOW have been made at a number of Xsep locations. Results at Xsep $=-9$ " are plotted on Fig. 10; CFD and wind tunnel data for Xsep values of -20 " and -44 " are presented in Figs. 11 and 12. Where CFD configurations match with wind tunnel data points, agreement is excellent; simulations at Zsep equiv $<0$ yield relatively small variations in $C_{N}, C_{m}$ and $C_{A}$. An examination of the computed flowfields shows that for these cases, the adapter lip remains in the flyer wake. This behavior supports the mapping of Asep and Zsep into Zsepequiv, and indicates the need to revise the current extrapolation methodology used by the aerodynamic database.

In addition to investigating force and moment variation with vertical offset, CFD runs were made to define the limit of Xsep for which the HXRV exhibited aerodynamic interference from the adapter. ${ }^{25}$ The maximum horizontal separation tested in the AEDC and 20" Mach 6 tunnels was Xsep $=-44$ ". While the

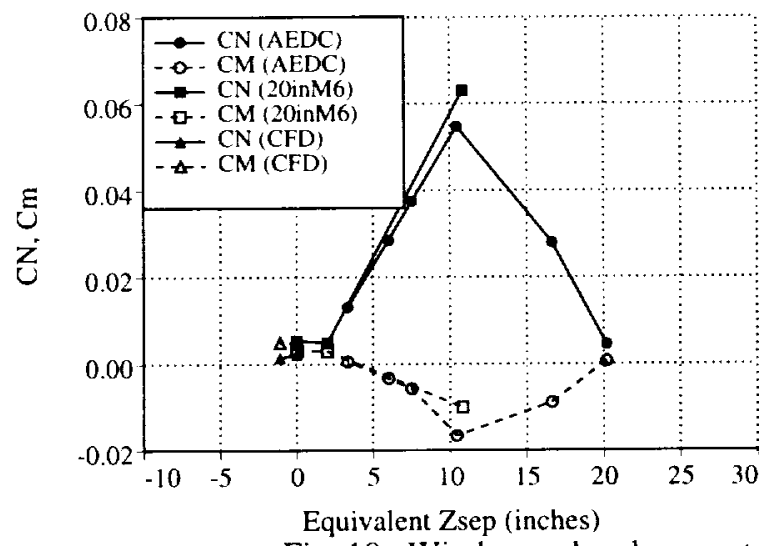

coefficient variations displayed at smaller separation distances in Fig. 7 are small, these values are significantly different from flyer-alone values. In order to define the horizontal extent of adapter interference, a series of runs were made with SAMcfd at Xsep values of $-60,-70,-80$, and -104 inches, all at $\alpha=0$ and zero Asep and Zsep. Plotted in Fig. 13, these results show that $C_{N}, C_{m}$ and $C_{A}$ reach their interference-free values by Xsep $=-70^{\prime \prime}$

It should be noted that the process of extrapolating the aerodynamic database is critical, since extrapolation generally results in large uncertainties. The database and uncertainty levels serve as input to the dynamic trajectory simulations, which use Monte Carlo variations of the aerodynamic forces, initial and other conditions to evaluate likelihood of the research vehicle successfully separating from the booster and arriving at the scramjet test condition. An improvement in the extrapolation methodology thus not only improves the fidelity of the aerodynamic database, but allows a reduction in the associated uncertainties and an improvement in the Monte Carlo simulations as well.

\section{Wind Tunnel vs. Flight Environments}

A number of issues arise when extrapolating wind tunnel-measured data to flight conditions. For the Hyper-X stage separation, these effects can be broadly categorized into differences in geometry and flow conditions.

Two differences related to geometry exist between the flight article and wind tunnel models. The primary geometry difference is the presence or absence of the wind tunnel model support. In the stage separation tests, the flyer was supported by a vertical blade connected to the top of the flyer. Flyer-alone tests in the 20" Mach 6 tunnel used either a sting or a blade mount, and considerable testing was done with a dummy blade or a dummy sting (as appropriate) to generate deltas due to the support method. Flyer-alone CFD runs were made with and without a blade, as were separation cases at several values of Xsep. These are

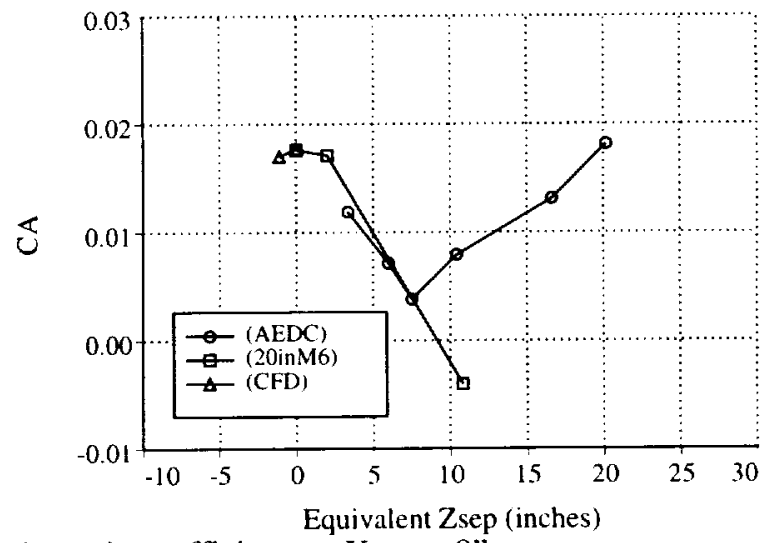

Fig. 10. Wind tunnel and computed aerodynamic coefficients at Xsep $=-9^{\prime \prime}$. 

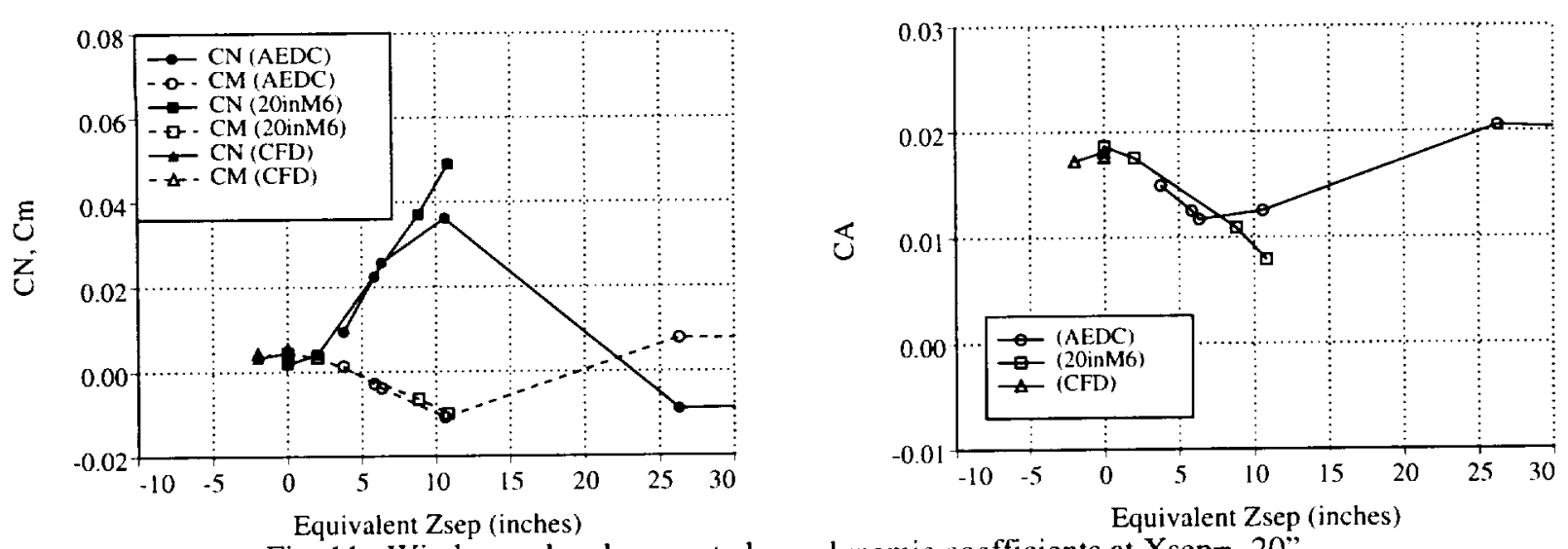

Fig. 11. Wind tunnel and computed acrodynamic coefficients at Xsep $=-20$ ".
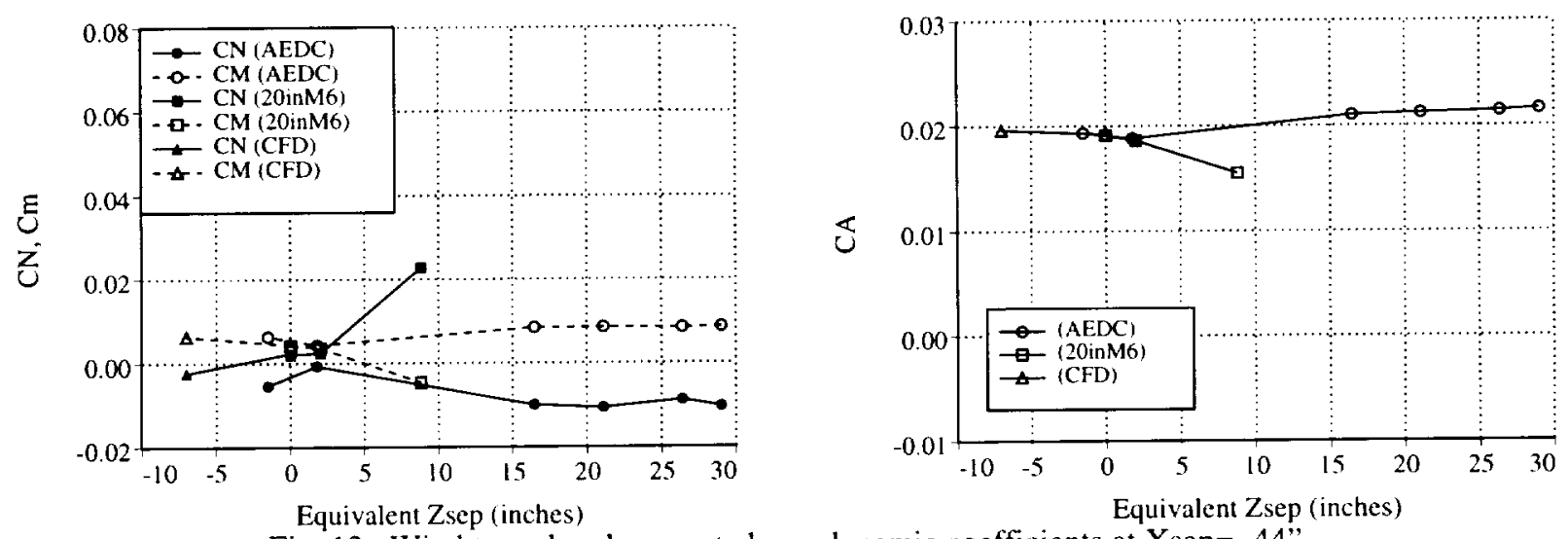

Fig. 12. Wind tunnel and computed aerodynamic coefficients at Xsep=-44".
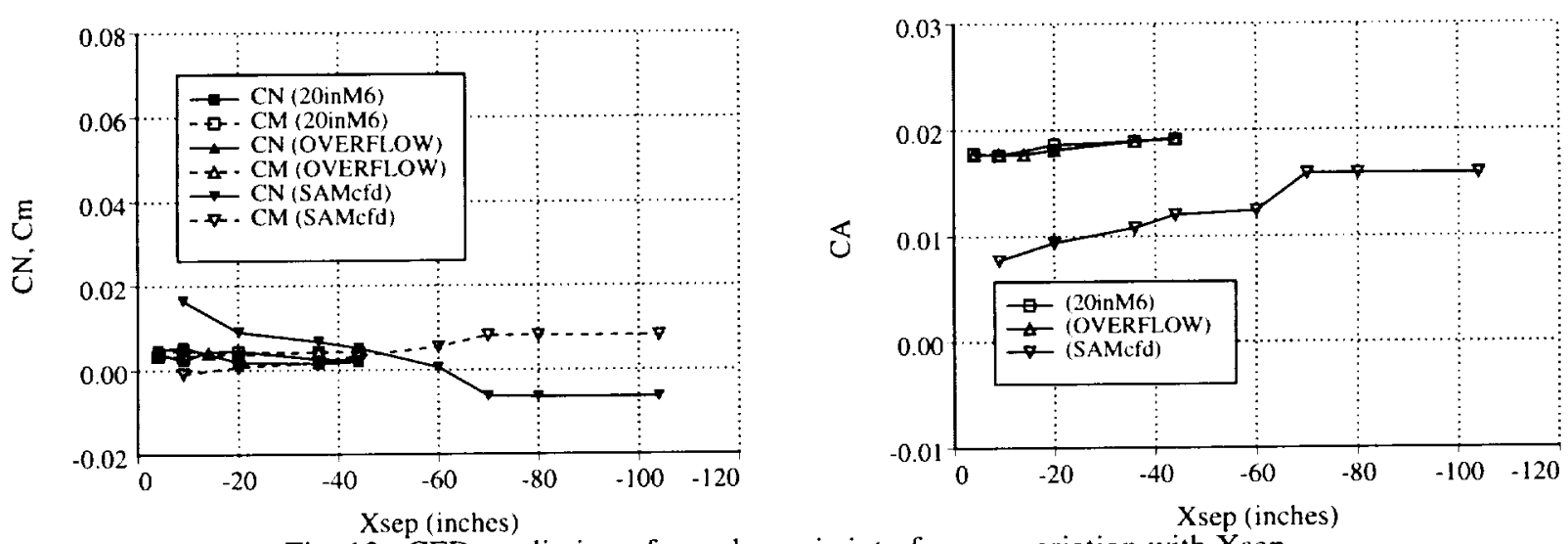

Fig. 13. CFD prediction of aerodynamic interference variation with Xsep.

presented in Table 1, and show that the blade interference, while significant, is relatively independent of Xsep. The use of a blade increment that is independent of Xsep is therefore justified.

The second geometry difference between wind tunnel and flight is the seal on the front of the adapter, which mates to the bottom and side of the engine cowl. The flight definition for this piece of geometry has changed several times, and due to its thinness was unsuitable for model scale testing. As a result, the wind tunnel seal geometry is thicker with a blunt leading edge. While the current flight seal design has not been simulated, both the wind tunnel design and an earlier thin seal design have. (These three geometries are compared in Fig. 14.) Differences in integrated forces between these cases were small: at Xsep $=-4$ ", $\Delta \mathrm{C}_{\mathrm{v}}=0.0016, \Delta \mathrm{C}_{\mathrm{m}}=-0.0006$, and $\Delta \mathrm{C}_{\mathrm{A}}=-0.0001$. 


\begin{tabular}{cllll}
\hline & Xsep (inches) & $\Delta \mathrm{C}_{\mathrm{N}}$ & $\Delta \mathrm{C}_{\mathrm{m}}$ & $\Delta \mathrm{C}_{\mathrm{A}}$ \\
\hline OVERFLOW & Flyer alone & 0.0058 & -0.0022 & -0.0005 \\
(turbulent) & -44 & 0.0058 & -0.0022 & -0.0003 \\
& -4 & 0.0052 & -0.0020 & -0.0001 \\
SAMcfd (inviscid) & Flyer alone & 0.0084 & -0.0031 & -0.0004 \\
(Ref. 26) & -44 & 0.0085 & -0.0031 & -0.0004 \\
& -9 & 0.0055 & -0.0019 & -0.0002 \\
20" Mach 6 WT & Flyer alone & 0.0083 & -0.0026 & -0.0010 \\
\hline
\end{tabular}

Table 1. Comparison of CFD and experimental blade increments.

Differences in flow conditions between wind tunnel and flight include freestream Mach number, Reynolds number, laminar vs. turbulent flow, and isothermal vs. adiabatic walls. One clear difference in flow conditions between wind tunnel and flight is the Mach number. Flight is at Mach 7, while wind tunnel tests used to derive the aerodynamic database were conducted at Mach 6. Wind tunnel tests at Mach 6 and 10 , and inviscid SAMcfd simulations indicate little sensitivity in aerodynamic coefficients in this Mach number range.

Wind tunnel tests on stage separation configurations were tripped to force turbulent flow, in order to minimize blade interference effects. However, flyer-alone tests were run without boundary layer trips. Tests of this configuration at a range of Reynolds numbers showed very little change in axial force, indicating the possibility of transitional flow. Comparison of laminar and turbulent CFD simulations for both research vehicle-alone and stage separation configurations show an increment in axial force coefficient of 0.0014 to 0.0020 , but no significant change in pitching moment or normal force. To investigate Reynolds number and wall temperature effects independent of boundary layer turbulence, a flyer-alone calculation was made at Mach 6, with laminar flow at flight Reynolds number and a fixed wall temperature of $1,500^{\circ} \mathrm{R}$. Aerodynamic coefficients for this simulation are included in Fig. 3, and also show no change in normal force and pitching moment. These analyses support the assumption that

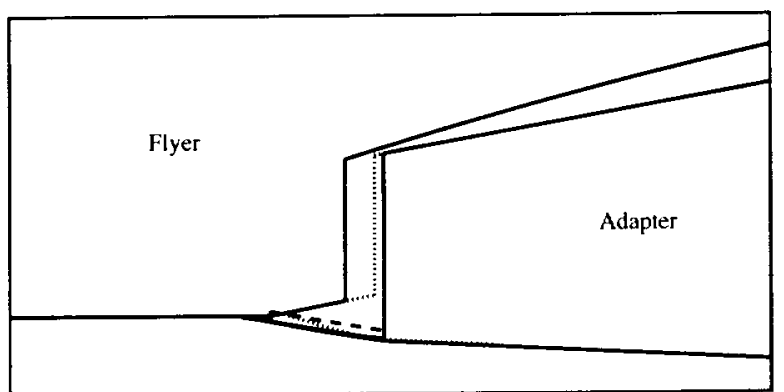

Fig. 14. Schematic of earlier thin seal (solid), wind tunnel seal (dashed), and current flight design (dotted). turbulence, Reynolds number, and wall temperature conditions affect only axial force.

\section{Conclusions}

The stage separation event for the Hyper-X research program, where the research vehicle separates from the nose of the Orbital Sciences Pegasus booster, is a complex aerodynamic maneuver. While the aerodynamic database used for evaluating the separation trajectory under a variety of scenarios is based on wind tunnel-derived force and moment coefficients, questions have arisen about several aerodynamic aspects of the separation. CFD has played a role in answering these questions and reducing the risks associated with this part of the flight. Specifically, CFD simulations have been used to:

- Confirm that initial aerodynamic transients of the separation are of short duration;

- Illustrate the appropriateness of quasi-steady modeling of the separation aerodynamics;

- Highlight the physics behind nonlinear variation of the aerodynamic coefficients with vertical clearance, and provide a different methodology for extending the database into the region of the nominal trajectory;

- Determine the horizontal extent of aerodynamic interference from the adapter;

- Confirm methodology for gauging model support interference; and

- Support assumptions about the effects of geometric and flow differences from wind tunnel to flight conditions.

Further, the study of these issues provides information on the usefulness and accuracy available from both inviscid and viscous CFD simulations, establishing an experience base for the development of follow-on vehicles.

\section{Acknowledgements}

The authors would like to acknowledge the many fruitful discussions with Mr. Walter C. Engelund of the NASA Langley Vehicle Analysis Branch and Mr. Charles E. Cockrell, Jr., of the Hypersonic Airbreathing 
Propulsion Branch. Dr. Scott D. Holland and Mr. William C. Woods of the Langley Aerothermodynamics Branch provided critical information on the wind tunnel tests used for comparison. Support from the Hyper-X Program Office and Mr. David E. Reubush, Hyper-X Stage Separation Manager, is gratefully acknowledged.

Computer time for this effort was supplied by the NASA Numerical Aerodynamic Simulation (NAS) Facility at NASA Ames Research Center, CA, and the Department of Defense NAVO Major Shared Resource Center at Stennis Space Center, MS.

\section{References}

1. D.E. Reubush, "Hyper-X Stage SeparationBackground and Status," AIAA 99-4818, Nov. 1999.

2. W. Woods and S. Holland, "Hyper-X Stage Separation Wind Tunnel Test Program," AIAA 2000-4008, Aug. 2000.

3. J.L. Pao, "Pre-Test SAMcfd Euler Analysis for Hyper-X Stage Separation AEDC Tests," HX-432, TN 99-289, Hyper-X Program Office, NASA Langley Research Center, Mar. 2000

4. W. Engelund, S. Holland, and C. Cockrell, Jr., "Aerodynamic Database Development for the Hyper-X Airframe Integrated Scramjet Propulsion Experiment," AIAA 2000-4006, Aug. 2000.

5. C. Cockrell, Jr., W. Engelund, R. Bittner, A. Dilley, T. Jentink, and A. Frendi, "Integrated AeroPropulsive CFD Methodology for the Hyper-X Flight Experiment," AIAA 2000-4010, Aug. 2000.

6. R. Lohner, "Three-Dimensional Fluid-Structure Interaction Using a Finite Element Solver and Adaptive Remeshing," Computing Systems in Engineering, Vol. 1, 1990.

7. L.W. Spradley, "Data Transfer Between SAMcfd and NASTRAN," Phase III Final Report, NASA SBIR NAS1-97106, ResearchSouth Inc., Feb. 1998.

8. D.C. Jespersen, T.H. Pulliam, and P.G. Buning, "Recent Enhancements to OVERFLOW," AIAA 97-0644, Jan. 1997.

9. P.G. Buning, D.C. Jespersen, T.H. Pulliam, G.H. Klopfer, W.M. Chan, J.P. Slotnick, S.E. Krist, and K.J. Renze, "OVERFLOW User's Manual, Version 1.8m," NASA Langley Research Center, Jan. 2000.

10. W.M. Chan and R.J. Gomez, "Advances in Automatic Overset Grid Generation Around Surface Discontinuities," AIAA 99-3303, July 1999.

11. N.E. Suhs and R.W. Tramel, "PEGSUS 4.0 User's Manual," AEDC-TR-91-8, Arnold Engineering Development Center, Arnold AFB, TN, Nov. 1991.
12. F.M. White, Viscous Fluid Flow, McGraw-Hill, Inc., New York, 1974, p. 498.

13. S.C. Sommer and B.J. Short, "Free-Flight Measurements of Turbulent Boundary Layer Skin Friction in the Presence of Severe Aerodynamic Heating at Mach Numbers from 2.8 to 7.0," Journal of the Aeronautical Sciences, Vol. 23, No. 6, June 1956.

14. P.R. Spalart and S.R. Allmaras, "A One-Equation Turbulence Model for Aerodynamic Flows," La Recherche Aerospatiale, No. 1, 5-21, 1994.

15. S. Holland, W. Woods, and W. Engelund, "Hyper$X$ Research Vehicle (HXRV) Experimental Aerodynamics Test Program Overview," AIAA 2000-4011, Aug. 2000.

16. A.D. Dilley and J.L. Pao, "Mach 7 Time Accurate, Inviscid Computations with a Fixed 2D Cavity," HX-432, TN 98-204, Hyper-X Program Office, NASA Langley Research Center, June 1998.

17. S.L. Krist, R.T. Biedron, and C.L. Rumsey, "CFL3D User's Manual (Version 5.0)," NASA TM-1998-208444, June 1998.

18. T.-C. Wong, "Two-Dimensional, Time-Accurate, Viscous Analysis with a Fixed Cavity Geometry," HX-432, TN 00-313, Hyper-X Program Office, NASA Langley Research Center, May 2000.

19. R. Meakin and A. Wissink, "Unsteady Aerodynamic Simulation of Static and Moving Bodies Using Scalable Computers," AIAA 993302, June 1999.

20. R. Meakin, "The Chimera Method of Simulation for Unsteady Three-dimensional Viscous Flow," Computational Fluid Dynamics Review 1995 , M. Hafez and K. Oshima, eds, John Wiley, 1995.

21. R. Meakin and N. Suhs, "Unsteady Aerodynamic Simulation of Multiple Bodies in Relative Motion," AIAA 89-1996, June 1989.

22. X. Luo and O. Baysal, "Computational Simulation of Hypersonic Vehicle Separation from its Booster," AIAA 99-4807, Nov. 1999.

23. T. Cvrlje, "Unsteady Separation of a Two-Stage Hypersonic Vehicle," AIAA 99-3412, June 1999.

24. W.A. Roettker, "Hyper-X Aerodynamics and Propulsion Reference Systems," HX-241, Hyper-X Program Office, NASA Langley Research Center, Apr. 1997.

25. J.L. Pao, "Interference Effects of the Fixed Jaw Adapter on the Hyper-X Research Vehicle Aerodynamics," HX-432, TN 99-249, Hyper-X Program Office, NASA Langley Research Center, Jan. 2000.

26. J.L. Pao, "Blade Interference Effects on the HyperX Research Vehicle Aerodynamics," HX-432, TN 00-293, Hyper-X Program Office, NASA Langley Research Center, May 2000. 